\title{
Problems of welding in shipbuilding - an analytic-numerical assessment of the thermal cycle in Haz with three dimensional heat source models in agreement with modelling rules
}

\author{
Part II \\ An analytical assessment of thermal cycle \\ by used C-I-N and D-E heat sources models
}

Eugeniusz Ranatowski, Prof

University of Technology and Life Science, Bydgoszcz

\section{ABSTRACT}

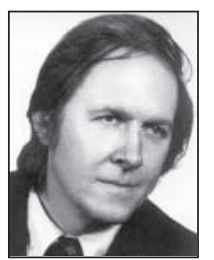

This part is continuation of PART I. The basis of this analytic solution are the FourierKirchhoff partial differential equation with appropriated boundary conditions. For a plate with optional thickness, the radiative heat transfer on both surfaces is taken into account. It is assumed that moving C-I-N or D-E heat sources during a very short period of time, generate an impulse of energy inducing an instantaneous thermal field in the plate area and the analytic solution is received by used Fourier transformation. These fields are being continuously summed up to obtain resultant thermal field $\left(T=\int_{0}^{t} d T\left(t^{\prime}\right)\right)$. Finally, the temperature fields generated by $C-I-N$ and D-E heat sources in both stationary and moving co-ordinates systems are established.

Keywords: welding; shipbuilding; welding in shipbuilding; thermal cycle; heat affected zone; heat source model

LINEAR ANALYTICAL HEAT FLOW

SOLUTION FOR A PLATE WITH

OPTIONAL THICKNESS AND RADIATIVE

HEAT TRANSFER ON SURFACES BY C-I-N HEAT SOURCE MODEL

Energy heat transport in HAZ is mainly progressed by thermal conduction and can be described by $\mathrm{F}-\mathrm{K}$ partial differential equation.

Here is the way how the solution for temporary temperature fields is received. The integral transformation method is being used.

HS power input in volume showed by eq. (12) - part I in impulse form is described as:

$$
\begin{gathered}
\mathrm{q}_{\mathrm{v}}=\frac{\mathrm{Q} \cdot \delta(\mathrm{t})}{\pi \cdot\left[1-\exp \left(-\mathrm{K}_{\mathrm{z}} \cdot \mathrm{s}\right)\right]} \cdot \mathrm{k} \cdot \mathrm{K}_{\mathrm{z}} \cdot \\
\cdot \exp \left[-\mathrm{k}\left(\mathrm{x}^{2}+\mathrm{y}^{2}\right)-\mathrm{K}_{\mathrm{z}} \cdot \mathrm{z}\right] \cdot[1-\mathrm{u}(\mathrm{z}-\mathrm{s})]
\end{gathered}
$$

or

$$
\begin{gathered}
\mathrm{q}_{\mathrm{v}}=\mathrm{q}_{\mathrm{vMAX}} \cdot \delta(\mathrm{t}) \cdot \\
\cdot \exp \left[-\mathrm{k}\left(\mathrm{x}_{0}^{2}+\mathrm{y}_{0}^{2}\right)-\mathrm{K}_{\mathrm{z}} \cdot \mathrm{z}_{0}\right] \cdot[1-\mathrm{u}(\mathrm{z}-\mathrm{s})]
\end{gathered}
$$

Putting it into F-K equation the following form is received $^{1}$ :

$$
\frac{\partial^{2} \mathrm{~T}}{\partial \mathrm{x}_{0}^{2}}+\frac{\partial^{2} \mathrm{~T}}{\partial \mathrm{y}_{0}^{2}}+\frac{\partial^{2} \mathrm{~T}}{\partial \mathrm{z}_{0}^{2}}+\frac{\mathrm{q}_{\mathrm{vMAX}} \cdot \delta(\mathrm{t})}{\lambda}
$$

$$
\cdot[1-\mathrm{u}(\mathrm{z}-\mathrm{s})] \cdot \exp \left[-\mathrm{k}\left(\mathrm{x}_{0}^{2}+\mathrm{y}_{0}^{2}\right)-\mathrm{K}_{\mathrm{z}} \cdot \mathrm{z}_{0}\right]=\frac{1}{\mathrm{a}} \frac{\partial \mathrm{T}}{\partial \mathrm{t}}
$$

Boundary conditions:

$$
\begin{aligned}
& \mathrm{T}\left(\mathrm{x}_{0}, \mathrm{y}_{0}, \mathrm{z}_{0}, \mathrm{t}=0\right)=\mathrm{T}_{0}=0 \\
& \frac{\partial \mathrm{T}}{\partial \mathrm{x}_{0}}=0 \text { when } \mathrm{x}_{0} \rightarrow \infty, \mathrm{x}_{0} \rightarrow-\infty \\
& \frac{\partial \mathrm{T}}{\partial \mathrm{y}_{0}}=0 \text { when } \mathrm{y}_{0} \rightarrow \infty, \mathrm{y}_{0} \rightarrow-\infty \\
& \lambda \cdot \frac{\partial \mathrm{T}}{\partial \mathrm{z}_{0}}=\alpha_{0} \cdot \mathrm{T} \text { when } \mathrm{z}_{0}=0 \\
& \lambda \cdot \frac{\partial \mathrm{T}}{\partial \mathrm{z}_{0}}=\alpha_{0} \cdot \mathrm{T} \text { when } \mathrm{z}_{0}=\mathrm{g}
\end{aligned}
$$


Graphic interpretation of the above conditions is shown in Fig. 1.

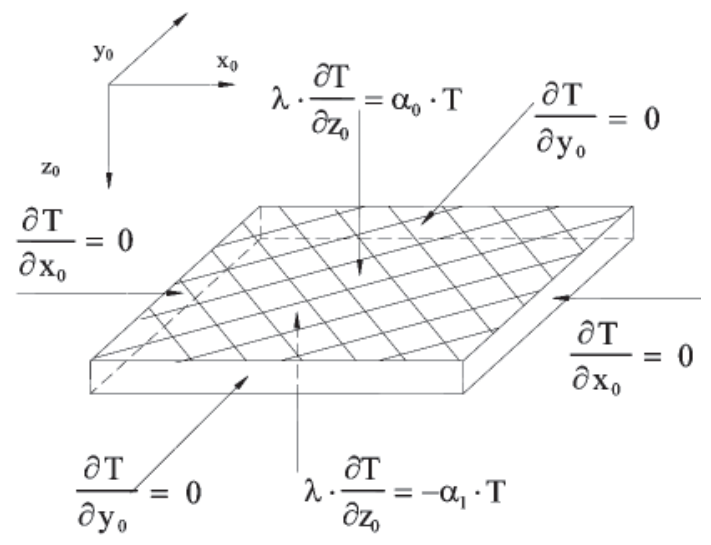

Fig. 1. Graphic interpretation of boundary conditions

The $\mathrm{x}_{0}, \mathrm{y}_{0}$ and $\mathrm{z}_{0}$ variable transformations for equation (3) are executed with use the classic Fourier transformation. Transformation module for $\mathrm{x}_{0}$ is described $\mathrm{as}^{2}$ :

$$
\mathrm{K}\left(\mathrm{r}, \mathrm{x}_{0}\right)=\exp \left(\mathrm{i} \cdot \mathrm{p} \cdot \mathrm{x}_{0}\right)
$$

The integration range is $(-\infty,+\infty)$. The equation (3) after multiplying by (4) looks like:

$$
\begin{array}{r}
\int_{-\infty}^{+\infty} \frac{\partial^{2} \mathrm{~T}}{\partial \mathrm{x}_{0}^{2}} \cdot \mathrm{e}^{\mathrm{i} \cdot \mathrm{p} \cdot \mathrm{x}_{0}} \mathrm{dx_{0 }}+\int_{-\infty}^{+\infty} \frac{\partial^{2} \mathrm{~T}}{\partial \mathrm{y}_{0}^{2}} \cdot \mathrm{e}^{\mathrm{i} \cdot \mathrm{p} \cdot \mathrm{x}_{0}} \mathrm{dx_{0 }}+ \\
+\int_{-\infty}^{+\infty} \frac{\partial^{2} \mathrm{~T}}{\partial \mathrm{z}_{0}^{2}} \cdot \mathrm{e}^{\mathrm{i} \cdot \mathrm{p} \cdot \mathrm{x}_{0}} \mathrm{dx_{0 }}+\int_{-\infty}^{+\infty} \frac{\partial^{2} \mathrm{~T}}{\partial \mathrm{x}_{0}^{2}} \cdot \frac{\mathrm{q}_{\mathrm{vMAX}} \cdot \delta(\mathrm{t})}{\lambda} .
\end{array}
$$

$\cdot[1-\mathrm{u}(\mathrm{z}-\mathrm{s})] \cdot \exp \left[-\mathrm{k}\left(\mathrm{x}_{0}^{2}\right)-\mathrm{K}_{\mathrm{z}} \cdot \mathrm{z}_{0}\right] \mathrm{dx} \mathrm{x}_{0}=\int_{-\infty}^{+\infty} \frac{1}{\mathrm{a}} \frac{\partial \mathrm{T}}{\partial \mathrm{t}} \cdot \mathrm{dx} \mathrm{x}_{0}$

Transforming operation:

$$
\begin{gathered}
\int_{-\infty}^{+\infty} \frac{\partial^{2} \mathrm{~T}}{\partial \mathrm{x}_{0}{ }^{2}} \cdot \mathrm{e}^{\mathrm{i} \cdot \mathrm{p} \cdot \mathrm{x}_{0}} \mathrm{dx_{0 }}= \\
=\left.\frac{\partial \mathrm{T}}{\partial \mathrm{x}_{0}} \cdot \mathrm{e}^{\mathrm{i} \cdot \mathrm{p} \cdot \mathrm{x}_{0}}\right|_{-\infty} ^{+\infty}-\mathrm{i} \cdot \mathrm{p} \cdot \int_{-\infty}^{+\infty} \frac{\partial \mathrm{T}}{\partial \mathrm{x}_{0}} \cdot \mathrm{e}^{\mathrm{i} \cdot \mathrm{p} \cdot \mathrm{x}_{0}} \mathrm{dx_{0 }}= \\
=\left.\frac{\partial \mathrm{T}}{\partial \mathrm{x}_{0}} \cdot \mathrm{e}^{\mathrm{i} \cdot \mathrm{p} \cdot \mathrm{x}_{0}}\right|_{-\infty} ^{+\infty}-\mathrm{i} \cdot \mathrm{p} \cdot\left(\left.\mathrm{T} \cdot \mathrm{e}^{\mathrm{i} \cdot \mathrm{p} \cdot \mathrm{x}_{0}}\right|_{-\infty} ^{+\infty}-\mathrm{i} \cdot \mathrm{p} \cdot\right. \\
\left.\cdot \int_{-\infty}^{+\infty} \mathrm{T} \cdot \mathrm{e}^{\mathrm{i} \cdot \mathrm{p} \cdot \mathrm{x}_{0}} \mathrm{dx_{0 }}\right)=\left.\frac{\partial \mathrm{T}}{\partial \mathrm{x} \mathrm{x}_{0}} \cdot \mathrm{e}^{\mathrm{i} \cdot \mathrm{p} \cdot \mathrm{x}_{0}}\right|_{\mathrm{x}_{0}=\infty}-\frac{\partial \mathrm{T}}{\partial \mathrm{x}_{0}} \\
\left.\cdot \mathrm{e}^{\mathrm{i} \cdot \mathrm{p} \cdot \mathrm{x}_{0}}\right|_{\mathrm{x}_{0}=-\infty}-\left.\mathrm{i} \cdot \mathrm{p} \cdot \mathrm{T} \cdot \mathrm{e}^{\mathrm{i} \cdot \mathrm{p} \cdot \mathrm{x}_{0}}\right|_{\mathrm{x}_{0}=\infty}+ \\
+\left.\mathrm{i} \cdot \mathrm{p} \cdot \mathrm{T} \cdot \mathrm{e}^{\mathrm{i} \cdot \mathrm{p} \cdot \mathrm{x}_{0}}\right|_{\mathrm{x}_{0}=-\infty}+\mathrm{i}^{2} \cdot \mathrm{p}^{2} \cdot \\
\cdot \int_{-\infty}^{+\infty} \mathrm{T} \cdot \mathrm{e}^{\mathrm{i} \cdot \mathrm{p} \cdot \mathrm{x}_{0}} \mathrm{dx} \mathrm{x}_{0}=0-0-0+0+ \\
+\left(-\mathrm{p}^{2} \cdot \int_{-\infty}^{+\infty} \mathrm{T} \cdot \mathrm{e}^{\mathrm{i} \cdot \mathrm{p} \cdot \mathrm{x}_{0}} \mathrm{dx} \mathrm{x}_{0}\right)=-\mathrm{p}^{2} \cdot \overline{\mathrm{T}}
\end{gathered}
$$

Transforming operations for: $\mathrm{y}_{0}, \mathrm{z}_{0}$, and $\mathrm{t}$ variables.

These are obviously transformations for variables other than $x_{0}$, which simplifies the counting.
Therefore for $\mathrm{y}_{0}, \mathrm{z}_{0}$ variable we'll obtain

$$
\begin{gathered}
\int_{-\infty}^{+\infty} \frac{\partial^{2} \mathrm{~T}}{\partial \mathrm{y}_{0}^{2}} \cdot \mathrm{e}^{\mathrm{i} \cdot \mathrm{p} \cdot \mathrm{x}_{0}} \mathrm{dx_{0 }}= \\
=\frac{\partial^{2}}{\partial \mathrm{y}_{0}{ }^{2}} \int_{-\infty}^{+\infty} \mathrm{T} \cdot \mathrm{e}^{\mathrm{i} \cdot \mathrm{p} \cdot \mathrm{x}_{0}} \mathrm{dx_{0 }}=\frac{\partial^{2}}{\partial \mathrm{y}_{0}{ }^{2}} \overline{\mathrm{T}} \\
\int_{-\infty}^{+\infty} \frac{\partial^{2} \mathrm{~T}}{\partial \mathrm{z}_{0}{ }^{2}} \cdot \mathrm{e}^{\mathrm{i} \cdot \mathrm{p} \cdot \mathrm{x}_{0}} \mathrm{dx_{0 }}= \\
=\frac{\partial^{2}}{\partial \mathrm{z}_{0}{ }^{2}} \int_{-\infty}^{+\infty} \mathrm{T} \cdot \mathrm{e}^{\mathrm{i} \cdot \mathrm{p} \cdot \mathrm{x}_{0}} \mathrm{dx_{0 }}=\frac{\partial^{2}}{\partial \mathrm{z}_{0}{ }^{2}} \overline{\mathrm{T}}
\end{gathered}
$$

and for $\mathrm{t}$ variable:

$$
\int_{-\infty}^{+\infty} \frac{\partial \mathrm{T}}{\partial \mathrm{t}} \cdot \mathrm{e}^{\mathrm{i} \cdot \mathrm{p} \cdot \mathrm{x}_{0}} \mathrm{dx} \mathrm{x}_{0}=\frac{\partial}{\partial \mathrm{t}} \int_{-\infty}^{+\infty} \mathrm{T} \cdot \mathrm{e}^{\mathrm{i} \cdot \mathrm{p} \cdot \mathrm{x}_{0}} \mathrm{dx}_{0}=\frac{\partial}{\partial \mathrm{t}} \overline{\mathrm{T}}
$$

Transforming operation for:

$$
\begin{aligned}
& \frac{\mathrm{q}_{\mathrm{vMAX}} \cdot \delta(\mathrm{t})}{\lambda} \cdot[1-\mathrm{u}(\mathrm{z}-\mathrm{s})] \cdot \\
& \cdot \exp \left[-\mathrm{k}\left(\mathrm{x}_{0}^{2}+\mathrm{y}_{0}^{2}\right)-\mathrm{K}_{\mathrm{z}} \cdot \mathrm{z}_{0}\right]
\end{aligned}
$$

where:

$$
\mathrm{q}_{\mathrm{vMAX}}=\frac{\mathrm{k} \cdot \mathrm{K}_{\mathrm{z}} \cdot \mathrm{Q}}{\pi \cdot\left[1-\exp \left(-\mathrm{K}_{\mathrm{z}} \cdot \mathrm{s}\right)\right]}
$$

$$
\int_{-\infty}^{+\infty} \frac{\mathrm{q}_{\mathrm{vMAX}} \cdot \delta(\mathrm{t})}{\lambda} \cdot[1-\mathrm{u}(\mathrm{z}-\mathrm{s})]
$$$$
\cdot \exp \left[-\mathrm{k}\left(\mathrm{x}_{0}^{2}+\mathrm{y}_{0}^{2}\right)-\mathrm{K}_{\mathrm{z}} \cdot \mathrm{z}_{0}\right] \cdot \mathrm{e}^{\mathrm{i} \cdot \mathrm{p} \cdot \mathrm{x}_{0}} \mathrm{dx_{0 }}=
$$$$
=\sqrt{\frac{\pi}{\mathrm{k}}} \frac{\mathrm{q}_{\mathrm{vMAX}} \cdot \delta(\mathrm{t})}{\lambda} \cdot[1-\mathrm{u}(\mathrm{z}-\mathrm{s})] \text {. }
$$

$$
\cdot \exp \left[-\mathrm{k}\left(\frac{\mathrm{p}^{2}}{4 \mathrm{k}}+\mathrm{y}_{0}^{2}\right)-\mathrm{K}_{\mathrm{z}} \cdot \mathrm{z}_{0}\right]
$$

and finally after first transformation the following is received:

$$
-\mathrm{p}^{2} \cdot \overline{\mathrm{T}}+\frac{\partial^{2}}{\partial \mathrm{y}_{0}^{2}} \overline{\mathrm{T}}+\frac{\partial^{2}}{\partial \mathrm{z}_{0}^{2}} \overline{\mathrm{T}}+\sqrt{\frac{\pi}{\mathrm{k}}} \frac{\mathrm{q}_{\mathrm{vMAX}} \cdot \delta(\mathrm{t})}{\lambda} .
$$

$\cdot[1-\mathrm{u}(\mathrm{z}-\mathrm{s})] \cdot \exp \left[-\mathrm{k}\left(\frac{\mathrm{p}^{2}}{4 \mathrm{k}}+\mathrm{y}_{0}^{2}\right)-\mathrm{K}_{\mathrm{z}} \cdot \mathrm{z}_{0}\right]=\frac{1}{\mathrm{a}} \cdot \frac{\partial}{\partial \mathrm{t}} \overline{\mathrm{T}}$

Similarly like for $\mathrm{x}_{0}$ this classic Fourier transformation we will use for $\mathrm{y}_{0}$ transformation.

Transformation module for $\mathrm{y}_{0}$ is described as:

$$
\mathrm{K}\left(\mathrm{r}, \mathrm{y}_{0}\right)=\exp \left(\mathrm{i} \cdot \mathrm{p} \cdot \mathrm{y}_{0}\right)
$$

The integration range is $(-\infty,+\infty)$. The equation (6) after multiplying by (7) and transforming looks like:

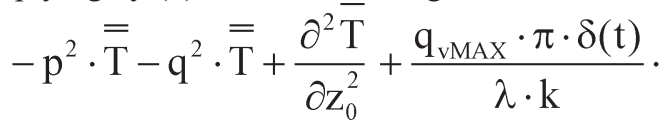

$[1-\mathrm{u}(\mathrm{z}-\mathrm{s})]) \exp \left(-\frac{\mathrm{p}^{2}}{4 \cdot \mathrm{k}}-\frac{\mathrm{q}^{2}}{4 \cdot \mathrm{k}}-\mathrm{K}_{\mathrm{z}} \cdot \mathrm{z}_{0}\right)=\frac{1}{\mathrm{a}} \frac{\partial \stackrel{=}{\partial \mathrm{T}}}{\mathrm{t}}$ 
Finally, transformation module for $\mathrm{z}_{0}$ is described as:

$$
\mathrm{K}\left(\mathrm{r}, \mathrm{z}_{0}\right)=\cos \left(\mathrm{r} \cdot \mathrm{z}_{0}\right)+\frac{\alpha_{0}}{\lambda \cdot \mathrm{r}} \cdot \sin \left(\mathrm{r} \cdot \mathrm{z}_{0}\right)
$$

Therefore:

$$
\begin{gathered}
-\left(\mathrm{p}^{2}+\mathrm{q}^{2}+\mathrm{r}^{2}\right) \cdot \overline{\overline{\mathrm{T}}}+\int_{0}^{\mathrm{g}} \frac{\mathrm{q}_{\mathrm{vMAX}} \cdot \pi \cdot \delta(\mathrm{t})}{\lambda \cdot \mathrm{k}} \\
\cdot[1-\mathrm{u}(\mathrm{z}-\mathrm{s})] \cdot \exp \left(-\frac{\mathrm{p}^{2}}{4 \cdot \mathrm{k}}-\frac{\mathrm{q}^{2}}{4 \cdot \mathrm{k}}-\mathrm{K}_{\mathrm{z}} \cdot \mathrm{z}_{0}\right) \\
\cdot \cos \left(\mathrm{r} \cdot \mathrm{z}_{0}\right)+\frac{\alpha_{0}}{\lambda \cdot \mathrm{r}} \cdot \sin \left(\mathrm{r} \cdot \mathrm{z}_{0}\right) \mathrm{d} \mathrm{z}_{0}=\frac{1}{\mathrm{a}} \frac{\partial \overline{\overline{\mathrm{T}}}}{\partial \mathrm{t}}
\end{gathered}
$$

and

$$
\begin{gathered}
-\left(\mathrm{p}^{2}+\mathrm{q}^{2}+\mathrm{r}^{2}\right) \cdot \overline{\overline{\mathrm{T}}}+\int_{0}^{\mathrm{g}} \frac{\mathrm{q}_{\mathrm{vMAX}} \cdot \pi \cdot \delta(\mathrm{t})}{\lambda \cdot \mathrm{k}} . \\
\cdot \exp \left(-\frac{\mathrm{p}^{2}}{4 \cdot \mathrm{k}}-\frac{\mathrm{q}^{2}}{4 \cdot \mathrm{k}}-\mathrm{K}_{\mathrm{z}} \cdot \mathrm{z}_{0}\right) \cdot \\
\cdot \int_{0}^{\mathrm{g}} \exp \left(-\mathrm{K}_{\mathrm{z}} \cdot \mathrm{z}_{0}\right) \cdot \cos \left(\mathrm{r} \cdot \mathrm{z}_{0}\right)+ \\
+\frac{\alpha_{0}}{\lambda \cdot \mathrm{r}} \cdot \sin \left(\mathrm{r} \cdot \mathrm{z}_{0}\right) \mathrm{d} \mathrm{z}_{0}=\frac{1}{\mathrm{a}} \frac{\partial \overline{\mathrm{T}}}{\partial \mathrm{t}}
\end{gathered}
$$

Let's find:

$$
\begin{aligned}
& \int_{0}^{\mathrm{s}} \exp \left(-\mathrm{K}_{\mathrm{z}} \cdot \mathrm{z}_{0}\right) \cdot \cos \left(\mathrm{r} \cdot \mathrm{z}_{0}\right)+\frac{\alpha_{0}}{\lambda \cdot \mathrm{r}} \text {. } \\
& \cdot \sin \left(\mathrm{r} \cdot \mathrm{z}_{0}\right) \mathrm{d} \mathrm{z}_{0}=\mathrm{D} \\
& \mathrm{D}=\exp \left(-\mathrm{K}_{\mathrm{z}} \cdot \mathrm{s}\right) \text {. } \\
& \cdot \frac{-\mathrm{K}_{\mathrm{z}} \cdot \cos (\mathrm{r} \cdot \mathrm{s}) \cdot \lambda \cdot \mathrm{r}+\mathrm{r}^{2} \cdot \sin (\mathrm{r} \cdot \mathrm{s}) \cdot}{\left(\mathrm{K}_{\mathrm{z}}{ }^{2}+\mathrm{r}^{2}\right) \cdot \lambda \cdot \mathrm{r}} \\
& \frac{\cdot \lambda-\alpha_{0} \mathrm{r} \cdot \cos (\mathrm{r} \cdot \mathrm{s})-\alpha_{0} \mathrm{~K}_{\mathrm{z}} \cdot \sin (\mathrm{r} \cdot \mathrm{s})}{1}+\frac{\mathrm{K}_{\mathrm{z}} \cdot \lambda+\alpha_{0}}{\left(\mathrm{~K}_{\mathrm{z}}{ }^{2}+\mathrm{r}^{2}\right) \cdot \lambda} \\
& \cot (r \cdot g)=\frac{\lambda^{2} r^{2}-\alpha_{0} \alpha_{1}}{\lambda \cdot r \cdot\left(\alpha_{0}+\alpha_{1}\right)} \\
& -\left(\mathrm{p}^{2}+\mathrm{q}^{2}+\mathrm{r}^{2}\right) \cdot \overline{\overline{\mathrm{T}}}+\frac{\mathrm{q}_{\mathrm{vMAX}} \cdot \pi \cdot \delta(\mathrm{t})}{\lambda \cdot \mathrm{k}} . \\
& \cdot \exp \left(-\frac{\mathrm{p}^{2}}{4 \cdot \mathrm{k}}-\frac{\mathrm{q}^{2}}{4 \cdot \mathrm{k}}\right) \cdot \mathrm{D}=\frac{1}{\mathrm{a}} \frac{\partial \overline{\mathrm{T}}}{\partial \mathrm{t}}
\end{aligned}
$$

The solution of (13) is Green function corresponding to differential operator:

$$
\frac{d}{d t}+a \cdot\left(p^{2}+q^{2}+r^{2}\right)
$$

The Green's functions solution is as follows:

$$
\begin{gathered}
\equiv=\frac{\mathrm{a} \cdot \mathrm{Q} \cdot \mathrm{K}_{\mathrm{z}} \cdot \mathrm{u}(\mathrm{t})}{\lambda \cdot\left(1-\exp \left(-\mathrm{K}_{\mathrm{z}} \cdot \mathrm{s}\right)\right.} \cdot \exp \left(-\frac{\mathrm{p}^{2}}{4 \cdot \mathrm{k}}-\frac{\mathrm{q}^{2}}{4 \cdot \mathrm{k}}\right) . \\
\cdot \mathrm{D} \cdot \exp \left[-\mathrm{a} \cdot\left(\mathrm{p}^{2}+\mathrm{q}^{2}+\mathrm{r}^{2}\right) \cdot \mathrm{t}\right]
\end{gathered}
$$

In order to obtain solution for $\mathrm{T}(\mathrm{x}, \mathrm{y}, \mathrm{z}, \mathrm{t})$ - the reverse transformations must be provided.

At first, reverse transformations with specified modules for " $\mathrm{x}_{0}$ " and " $\mathrm{y}_{0}$ " will be executed.

The reverse transformations modules are as follows:

$$
\begin{aligned}
& \mathrm{R}\left(\mathrm{p}, \mathrm{x}_{0}\right)=\frac{1}{2 \pi} \cdot \exp \left(-\mathrm{i} \cdot \mathrm{p} \cdot \mathrm{x}_{0}\right) \\
& \mathrm{R}\left(\mathrm{q}, \mathrm{y}_{0}\right)=\frac{1}{2 \pi} \cdot \exp \left(-\mathrm{i} \cdot \mathrm{q} \cdot \mathrm{y}_{0}\right)
\end{aligned}
$$

The integration range is $(-\infty,+\infty)$ for both: "p" and " $q$ " variables.

In turn, the equation (14) after multiplying by (16) and "p" retransformation looks like:

$$
\begin{gathered}
\overline{\mathrm{T}}=\frac{\mathrm{a} \cdot \mathrm{Q} \cdot \mathrm{K}_{\mathrm{z}} \cdot \mathrm{u}(\mathrm{t}) \cdot \mathrm{D}}{\sqrt{\pi} \cdot \lambda \cdot\left[1-\exp \left(-\mathrm{K}_{\mathrm{z}} \cdot \mathrm{s}\right)\right]} \cdot \\
\cdot \exp \left(-\frac{\mathrm{q}^{2}}{4 \cdot \mathrm{k}}-\mathrm{a} \cdot\left(\mathrm{q}^{2}+\mathrm{r}^{2}\right) \cdot \mathrm{t}-\frac{\mathrm{x}_{0}^{2}}{4 \cdot \mathrm{a} \cdot \mathrm{t}+1}\right)
\end{gathered}
$$

Then, the equation (17) after multiplying by (16) and "q" retransformation looks like:

$$
\begin{gathered}
\overline{\mathrm{T}}=\frac{\mathrm{Q} \cdot \mathrm{K}_{\mathrm{z}} \cdot \mathrm{k} \cdot \mathrm{u}(\mathrm{t})}{\pi \cdot \mathrm{c}_{\gamma} \cdot\left(1-\exp \left(-\mathrm{K}_{\mathrm{z}} \cdot \mathrm{s}\right) \cdot(1+4 \cdot \mathrm{a} \cdot \mathrm{t} \cdot \mathrm{k})\right.} . \\
\cdot \mathrm{D} \cdot \exp \left[-\mathrm{a} \cdot \mathrm{r}^{2} \cdot \mathrm{t}-\frac{\mathrm{k} \cdot\left(\mathrm{x}_{0}^{2}+\mathrm{y}_{0}{ }^{2}\right)}{1+4 \cdot \mathrm{a} \cdot \mathrm{t} \cdot \mathrm{k}}\right]
\end{gathered}
$$

where:

$$
\mathrm{c}_{\gamma}=\mathrm{c}_{\mathrm{p}} \cdot \rho
$$

The last transformation for " $\mathrm{z}_{0}$ " variable is defined as:

$$
\mathrm{T}=\sum_{\mathrm{i}=1}^{\infty} \overline{\mathrm{T}}_{\mathrm{i}} \mathrm{R}_{\mathrm{i}}\left(\mathrm{r}, \mathrm{z}_{0}\right)
$$

with $\mathrm{z}_{0}$ retransformation module:

$$
\mathrm{R}_{\mathrm{i}}\left(\mathrm{r}, \mathrm{z}_{0}\right)=\mathrm{B}_{\mathrm{i}} \cdot \mathrm{C}_{\mathrm{i}}
$$

and $B_{i}, C_{i}$, values are in agreement with (23a) and (23b).

Finally, HS impulse temperature field is:

$$
\begin{gathered}
\mathrm{T}=\frac{\mathrm{Q} \cdot \mathrm{K}_{\mathrm{z}} \cdot \mathrm{k} \cdot \mathrm{u}(\mathrm{t})}{\pi \cdot \mathrm{c}_{\gamma} \cdot\left(1-\exp \left(-\mathrm{K}_{\mathrm{z}} \cdot \mathrm{s}\right) \cdot(1+4 \cdot \mathrm{a} \cdot \mathrm{t} \cdot \mathrm{k})\right.} \\
\cdot \exp \left[-\frac{\mathrm{k} \cdot\left(\mathrm{x}_{0}^{2}+\mathrm{y}_{0}^{2}\right)}{1+4 \cdot \mathrm{a} \cdot \mathrm{t} \cdot \mathrm{k}}\right] . \\
\cdot \sum_{\mathrm{i}=1}^{\infty} \mathrm{B}_{\mathrm{i}} \mathrm{C}_{\mathrm{i}} \mathrm{D}_{\mathrm{i}} \cdot \exp \left(-\mathrm{a} \cdot \mathrm{r}^{2} \cdot \mathrm{t}\right)
\end{gathered}
$$

Total temperature distribution from moving HS can be achieved by summing HS impulse results on its movement path:

$$
T(t)=\int_{0}^{t} d T\left(t^{\prime}\right)
$$

Therefore, in stationary co-ordinates system:

$$
\begin{gathered}
\mathrm{dT}=\frac{\mathrm{Q} \cdot \mathrm{K}_{\mathrm{z}} \cdot \mathrm{k} \cdot \mathrm{u}(\mathrm{t})}{\pi \cdot \mathrm{c}_{\gamma} \cdot\left[1-\exp \left(-\mathrm{K}_{\mathrm{z}} \cdot \mathrm{s}\right) \cdot\left(1+4 \cdot \mathrm{a} \cdot\left(\mathrm{t}-\mathrm{t}^{\prime}\right) \cdot \mathrm{k}\right)\right]} \cdot \\
\cdot \exp \left[-\frac{\mathrm{k} \cdot\left[\left(\mathrm{x}_{0}-\mathrm{v} \cdot \mathrm{t}^{\prime}\right)^{2}+\mathrm{y}_{0}^{2}\right]}{1+4 \cdot \mathrm{a} \cdot\left(\mathrm{t}-\mathrm{t}^{\prime}\right) \cdot \mathrm{k}}\right] \sum_{\mathrm{i}=1}^{\infty} \mathrm{B}_{\mathrm{i}} \cdot \mathrm{C}_{\mathrm{i}} \cdot \mathrm{D}_{\mathrm{i}} \cdot \\
\cdot \exp \left(-\mathrm{a} \cdot \mathrm{r}^{2} \cdot\left(\mathrm{t}-\mathrm{t}^{\prime}\right)\right.
\end{gathered}
$$




$$
\begin{gathered}
\mathrm{T}=\int_{0}^{\mathrm{t}} \mathrm{dt} \frac{\mathrm{Q} \cdot \mathrm{K}_{\mathrm{z}} \cdot \mathrm{k} \cdot \mathrm{u}(\mathrm{t})}{\pi \cdot \mathrm{c}_{\gamma} \cdot\left[1-\exp \left(-\mathrm{K}_{\mathrm{z}} \cdot \mathrm{s}\right)\right] \cdot\left[1+4 \cdot \mathrm{a} \cdot\left(\mathrm{t}-\mathrm{t}^{\prime}\right) \cdot \mathrm{k}\right]} \cdot \\
\cdot \exp \left[-\frac{\mathrm{k} \cdot\left[\left(\mathrm{x}_{0}-\mathrm{v} \cdot \mathrm{t}^{\prime}\right)^{2}+\mathrm{y}_{0}^{2}\right]}{1+4 \cdot \mathrm{a} \cdot \mathrm{t} \cdot \mathrm{k}}\right] \cdot \sum_{\mathrm{i}=1}^{\infty} \mathrm{B}_{\mathrm{i}} \cdot \mathrm{C}_{\mathrm{i}} \cdot \mathrm{D}_{\mathrm{i}} \cdot \\
\cdot \exp \left[-\mathrm{a} \cdot \mathrm{r}^{2} \cdot\left(\mathrm{t}-\mathrm{t}^{\prime}\right)\right]
\end{gathered}
$$

where:

$$
\begin{gathered}
\mathrm{B}_{\mathrm{i}}=\cos \left(\mathrm{r}_{\mathrm{i}} \cdot \mathrm{z}_{0}\right)+\frac{\alpha_{0}}{\lambda \cdot \mathrm{r}_{\mathrm{i}}} \cdot \sin \left(\mathrm{r}_{\mathrm{i}} \cdot \mathrm{z}_{0}\right) \\
\mathrm{C}_{\mathrm{i}}=\frac{2 \cdot \mathrm{r}_{\mathrm{i}}^{2}}{\left(\frac{\alpha_{0}^{2}}{\lambda^{2}}+\mathrm{r}_{\mathrm{i}}^{2}\right) \cdot\left(\mathrm{g}+\frac{\alpha_{1} \cdot \lambda}{\alpha_{1}^{2}+\mathrm{r}_{\mathrm{i}}^{2} \cdot \lambda^{2}}\right)+\frac{\alpha_{0}}{\lambda}}
\end{gathered}
$$

$$
\mathrm{D}_{\mathrm{i}}=\exp \left(-\mathrm{K}_{\mathrm{z}} \cdot \mathrm{s}\right) \cdot \frac{\left[-\mathrm{K}_{\mathrm{z}} \cdot \cos \left(\mathrm{r}_{\mathrm{i}} \cdot \mathrm{s}\right) \cdot \lambda \cdot \mathrm{r}_{\mathrm{i}}+\mathrm{r}_{\mathrm{i}}^{2} \cdot \sin \left(\mathrm{r}_{\mathrm{i}} \cdot \mathrm{s}\right) \cdot\right.}{\left(\mathrm{K}_{\mathrm{z}}^{2}+\mathrm{r}_{\mathrm{i}}^{2}\right) \cdot \lambda \cdot \mathrm{r}_{\mathrm{i}}}
$$$$
\frac{\left.\cdot \lambda-\alpha_{0} \cdot \mathrm{r}_{\mathrm{i}} \cdot \cos \left(\mathrm{r}_{\mathrm{i}} \cdot \mathrm{s}\right)-\alpha_{0} \cdot \mathrm{K}_{\mathrm{z}} \cdot \sin \left(\mathrm{r}_{\mathrm{i}} \cdot \mathrm{s}\right)\right]}{1}+
$$

$$
+\frac{\mathrm{K}_{\mathrm{z}} \cdot \lambda+\alpha_{0}}{\left(\mathrm{~K}_{\mathrm{z}}^{2}+\mathrm{r}_{\mathrm{i}}^{2}\right) \cdot \lambda}
$$

$\mathrm{r}_{1}, \mathrm{r}_{2}, \mathrm{r}_{3} \ldots \mathrm{r}_{\mathrm{i}}$ are roots of :

$$
\operatorname{ctg}\left(r_{i} \cdot g\right)=\frac{\lambda^{2} \cdot r_{i}^{2}-\alpha_{0} \cdot \alpha_{1}}{\lambda \cdot r_{i} \cdot\left(\alpha_{0}+\alpha_{1}\right)}
$$

In moving co-ordinates system, $\mathrm{x}=\mathrm{x}_{0}-\mathrm{vt}, \mathrm{y}=\mathrm{y}_{0}, \mathrm{z}=\mathrm{z}_{0}$ (Fig. 1, part I):

$$
\begin{aligned}
& \mathrm{T}=\int_{0}^{\mathrm{t}} \mathrm{dt} \frac{\mathrm{Q} \cdot \mathrm{K}_{\mathrm{z}} \cdot \mathrm{k} \cdot \mathrm{u}(\mathrm{t})}{\pi \cdot \mathrm{c}_{\gamma} \cdot\left[1-\exp \left(-\mathrm{K}_{\mathrm{z}} \mathrm{s}\right)\right] \cdot\left[1+4 \cdot \mathrm{a} \cdot\left(\mathrm{t}-\mathrm{t}^{\prime}\right) \cdot \mathrm{k}\right]} . \\
& \cdot \exp \left[-\frac{\left.\mathrm{k} \cdot\left[\left(\mathrm{x}+\mathrm{v} \cdot\left(\mathrm{t}-\mathrm{t}^{\prime}\right)\right)^{2}\right)+\mathrm{y}^{2}\right]}{1+4 \cdot \mathrm{a} \cdot\left(\mathrm{t}-\mathrm{t}^{\prime}\right) \cdot \mathrm{k}}\right] \text {. } \\
& \cdot \sum_{\mathrm{i}=1}^{\infty} \mathrm{B}_{\mathrm{i}} \mathrm{C}_{\mathrm{i}} \mathrm{D}_{\mathrm{i}} \cdot \exp \left[-\mathrm{a} \cdot \mathrm{r}^{2} \cdot\left(\mathrm{t}-\mathrm{t}^{\prime}\right)\right] \\
& \mathrm{B}_{\mathrm{i}}=\cos \left(\mathrm{r}_{\mathrm{i}} \cdot \mathrm{z}\right)+\frac{\alpha_{0}}{\lambda \cdot \mathrm{r}_{\mathrm{i}}} \cdot \sin \left(\mathrm{r}_{\mathrm{i}} \cdot \mathrm{z}\right)
\end{aligned}
$$

where:

$\mathrm{C}_{\mathrm{i}}, \mathrm{D}_{\mathrm{i}}, \mathrm{r}_{\mathrm{i}}$ - values are represented by same equations as for stationary system.

The equations (23), (24) for assessment of the temperature fields in both stationary and moving coordinates systems have far more extended form than classical analytical solution of Rosenthal-Rykalin. Certainly it results from accepted heat source model C-I-N and characterising it parameters like: $\mathrm{Q}, \mathrm{k}, \mathrm{K}, \mathrm{u}(\mathrm{z}-\mathrm{s})$. Furthermore, important elements of received solution are such parameters as: $\mathrm{B}_{\mathrm{i}}, \mathrm{C}_{\mathrm{i}}, \mathrm{D}_{\mathrm{i}}$. Their values also depend on such physical parameters as: $\lambda, \alpha, \alpha$ and roots $r_{i}$ from equation (23d). However eq. (23), (24) have correct forms of solutions from mathematical and physical points of view but they have too compound mathematical form and they are hard for direct analytical account. Besides, analytical form of solution makes calculation impossible when taking into consideration non-linear form of thermal process under welding. So, abovementioned affirmations make necessity of modification of analytical solution. This is possible through employment of hybrid analytical-numerical method. Present analytical form of solution defined by equations (23), (24) makes also impossible direct employment of numeric method for solution of these equations and assessment of the temperature fields.

\section{LINEAR ANALYTICAL HEAT FLOW SOLUTION FOR PLATE WITH OPTIONAL THICKNESS AND RADIATIVE HEAT TRANSFER ON SURFACES BY USED D-E HEAT SOURCE MODEL}

Similarly are estimated temperature fields for D-E heat source model but in a little complicated manner. At the beginning we must establish two partial differential heat flow equations for two different "quadrants" of another ellipsoids (Fig. 5, part I) at the same boundary conditions-equations $(3 \mathrm{a}) \div(3 \mathrm{e})$ :

- $\quad x>0$, (Fig. 5, part I)

$$
\begin{array}{r}
\frac{\partial^{2} \mathrm{~T}}{\partial \mathrm{x}_{0}^{2}}+\frac{\partial^{2} \mathrm{~T}}{\partial \mathrm{y}_{0}^{2}}+\frac{\partial^{2} \mathrm{~T}}{\partial \mathrm{z}_{0}^{2}}+\frac{\mathrm{q}_{\mathrm{v}} \mathrm{f}_{\mathrm{f}}}{\lambda}=\frac{1}{\alpha} \frac{\partial \mathrm{T}}{\partial \mathrm{t}} \\
\mathrm{x}<0, \text { (fig. 5, part I) } \\
\frac{\partial^{2} \mathrm{~T}}{\partial \mathrm{x}_{0}^{2}}+\frac{\partial^{2} \mathrm{~T}}{\partial \mathrm{y}_{0}^{2}}+\frac{\partial^{2} \mathrm{~T}}{\partial \mathrm{z}_{0}^{2}}+\frac{\mathrm{q}_{\mathrm{v}} \mathrm{f}_{\mathrm{r}}}{\lambda}=\frac{1}{\alpha} \frac{\partial \mathrm{T}}{\partial \mathrm{t}}
\end{array}
$$

In stationary coordinate system the $\mathrm{x}_{\mathrm{o}}, \mathrm{y}_{\mathrm{o}}$ and $\mathrm{z}_{\mathrm{o}}$ variable transformations for equations (25a), (25b) are executed in agreement with Fourier transformation as follows:

a. for variable $x_{0}$ with transformation module $K\left(p, x_{0}\right)=e^{-i p x_{0}}$ we will receive:

$$
\begin{gathered}
-\mathrm{p}^{2} \overline{\mathrm{T}}+\frac{\partial^{2} \overline{\mathrm{T}}}{\partial \mathrm{y}_{0}^{2}}+\frac{\partial^{2} \overline{\mathrm{T}}}{\partial \mathrm{z}_{0}^{2}}+\frac{6 \mathrm{f}_{\mathrm{f}} \cdot \mathrm{Q} \cdot \delta(\mathrm{t})}{\pi \lambda \mathrm{bc}} . \\
\cdot \exp \left(\frac{-3 \mathrm{a}_{1}^{2}\left(\mathrm{y}_{0}^{2} \mathrm{c}^{2}+\mathrm{z}_{0}^{2} \mathrm{~b}^{2}\right)}{\mathrm{a}_{1}^{2} \mathrm{~b}^{2} \mathrm{c}^{2}}-\frac{\mathrm{p}^{2} \mathrm{a}_{1}^{2}}{12}\right)=\frac{1}{\alpha} \frac{\partial \overline{\mathrm{T}}}{\partial \mathrm{t}} \\
-\mathrm{x}_{0}<0 \\
-\mathrm{p}^{2} \overline{\mathrm{T}}+\frac{\partial^{2} \overline{\mathrm{T}}}{\partial \mathrm{y}_{0}^{2}}+\frac{\partial^{2} \overline{\mathrm{T}}}{\partial \mathrm{z}_{0}^{2}}+\frac{6 \mathrm{f}_{\mathrm{r}} \cdot \mathrm{Q} \cdot \delta(\mathrm{t})}{\pi \lambda \mathrm{bc}} . \\
\cdot \exp \left(\frac{-3 \mathrm{a}_{2}^{2}\left(\mathrm{y}_{0}^{2} \mathrm{c}^{2}+\mathrm{z}_{0}^{2} \mathrm{~b}^{2}\right)}{\mathrm{a}_{2}^{2} \mathrm{~b}^{2} \mathrm{c}^{2}}-\frac{\mathrm{p}^{2} \mathrm{a}_{2}^{2}}{12}\right)=\frac{1}{\alpha} \frac{\partial \overline{\mathrm{T}}}{\partial \mathrm{t}}
\end{gathered}
$$

b. for variable $\mathrm{y}_{0}$ with transformation module $\mathrm{K}\left(\mathrm{p}, \mathrm{x}_{0}\right)=\mathrm{e}^{-\mathrm{ipx_{0 }}}$ we will receive:

$$
\begin{aligned}
& -\mathrm{p}^{2} \overline{\overline{\mathrm{T}}}-\mathrm{q}^{2} \overline{\overline{\mathrm{T}}}+\frac{\partial^{2} \overline{\overline{\mathrm{T}}}}{\partial \mathrm{z}_{0}^{2}}+\frac{2 \sqrt{3} \mathrm{f}_{\mathrm{f}} \cdot \mathrm{Q} \cdot \delta(\mathrm{t})}{\sqrt{\pi} \lambda \mathrm{c}} \\
& \cdot \exp \left[-\frac{3 \mathrm{z}^{2}}{\mathrm{c}^{2}}-\frac{1}{12}\left(\mathrm{p}^{2} \mathrm{a}_{1}^{2}+\frac{1}{12} \mathrm{q}^{2} \mathrm{~b}^{2}\right)\right]=\frac{1}{\alpha} \frac{\partial \overline{\overline{\mathrm{T}}}}{\partial \mathrm{t}} \\
& -\quad \text { for } \mathrm{x}_{0}<0 \\
& -\mathrm{p}^{2} \overline{\overline{\mathrm{T}}}-\mathrm{q}^{2} \overline{\overline{\mathrm{T}}}+\frac{\partial^{2} \overline{\overline{\mathrm{T}}}}{\partial \mathrm{z}_{0}^{2}}+\frac{2 \sqrt{3} \mathrm{f}_{\mathrm{r}} \cdot \mathrm{Q} \cdot \delta(\mathrm{t})}{\sqrt{\pi} \lambda \mathrm{c}} \\
& \cdot \exp \left[-\frac{3 \mathrm{z}_{0}^{2}}{\mathrm{c}^{2}}-\frac{1}{12}\left(\mathrm{p}^{2} \mathrm{a}_{2}^{2}+\mathrm{q}^{2} \mathrm{~b}^{2}\right)\right]=\frac{1}{\alpha} \frac{\partial \overline{\overline{\mathrm{T}}}}{\partial \mathrm{t}}
\end{aligned}
$$


c. for variable $\mathrm{z}_{0}$ with transformation module $\mathrm{K}\left(\mathrm{r}, \mathrm{z}_{0}\right)=\operatorname{cosrz} \mathrm{z}_{0}+\frac{\alpha_{0}}{\lambda \mathrm{r}} \operatorname{sinr} \mathrm{z}_{0}$ we will receive:

- for $\mathrm{x}_{0}>0$

$$
\begin{aligned}
& \overline{\overline{\mathrm{T}}}\left(-\mathrm{p}^{2}-\mathrm{q}^{2}-\mathrm{r}^{2}\right)+\frac{2 \sqrt{3} \mathrm{f}_{\mathrm{f}} \cdot \mathrm{Q} \cdot \delta(\mathrm{t})}{\sqrt{\pi} \lambda \mathrm{c}} . \\
& \exp \left[-\frac{1}{12}\left(p^{2} a_{1}^{2}+q^{2} b^{2}\right)\right] \text {. } \\
& \cdot \int_{0}^{\mathrm{g}}\left[\cos \left(\mathrm{rz}_{0}\right)+\frac{\alpha \alpha_{0}}{\lambda \mathrm{r}} \sin \left(\mathrm{rz}_{0}\right)\right] \cdot \mathrm{e}^{-\frac{3 \mathrm{z}_{0}^{2}}{\mathrm{c}^{2}}} \mathrm{dz_{0 }}=\frac{1}{\alpha} \frac{\partial \overline{\overline{\mathrm{T}}}}{\partial \mathrm{t}} \\
& \text { - } \text { for } \mathrm{x}_{0}<0 \\
& \overline{\overline{\bar{T}}}\left(-\mathrm{p}^{2}-\mathrm{q}^{2}-\mathrm{r}^{2}\right)+\frac{2 \sqrt{3} \mathrm{f}_{\mathrm{r}} \cdot \mathrm{Q} \cdot \delta(\mathrm{t})}{\sqrt{\pi} \lambda \mathrm{c}} \text {. } \\
& \cdot \exp \left[-\frac{1}{12}\left(p^{2} a_{2}^{2}+q^{2} b^{2}\right)\right] \text {. } \\
& \cdot \int_{0}^{\mathrm{g}}\left[\cos \left(\mathrm{rz}_{0}\right)+\frac{\alpha_{0}}{\lambda \mathrm{r}} \sin \left(\mathrm{rz}_{0}\right)\right] \cdot \mathrm{e}^{-\frac{3 \mathrm{z}_{0}^{2}}{\mathrm{c}^{2}}} \mathrm{dz_{0 }}=\frac{1}{\alpha} \frac{\partial \overline{\overline{\mathrm{T}}}}{\partial \mathrm{t}}
\end{aligned}
$$

During the Fourier transformation for the , $\mathrm{z}_{0}$ " variable it appears in eq. (28a) and (28b) the integral:

$$
\int_{0}^{\mathrm{g}}\left(\cos \left(\mathrm{rz}_{0}\right)+\frac{\alpha_{0}}{\lambda \mathrm{r}} \sin \left(\mathrm{rz}_{0}\right)\right) \cdot \exp \left(-\frac{3 \mathrm{z}_{0}^{2}}{\mathrm{c}^{2}}\right) \mathrm{dz_{0 }}
$$

The above integral can't be solved immediately but an algorithm can be used to obtain a satisfactory approach. In order to obtain the solution, the function $\exp \left(-3 \mathrm{z}_{0}^{2} / \mathrm{c}^{2}\right)$ may be written ${ }^{1}$ :

$$
\begin{gathered}
\exp \left(-\frac{3 z_{0}^{2}}{c^{2}}\right)=1+\sum_{n=1}^{\text {nlast }} \frac{\left(-\frac{3}{c^{2}} z^{2}\right)^{n}}{\left[\prod_{n=1}^{m}(n)\right]}= \\
=\operatorname{approx}(z, c, \text { nlast })
\end{gathered}
$$

In particular, nlast may be small when calculating for thin plates with quite large z-semi axis, see Fig. 2.
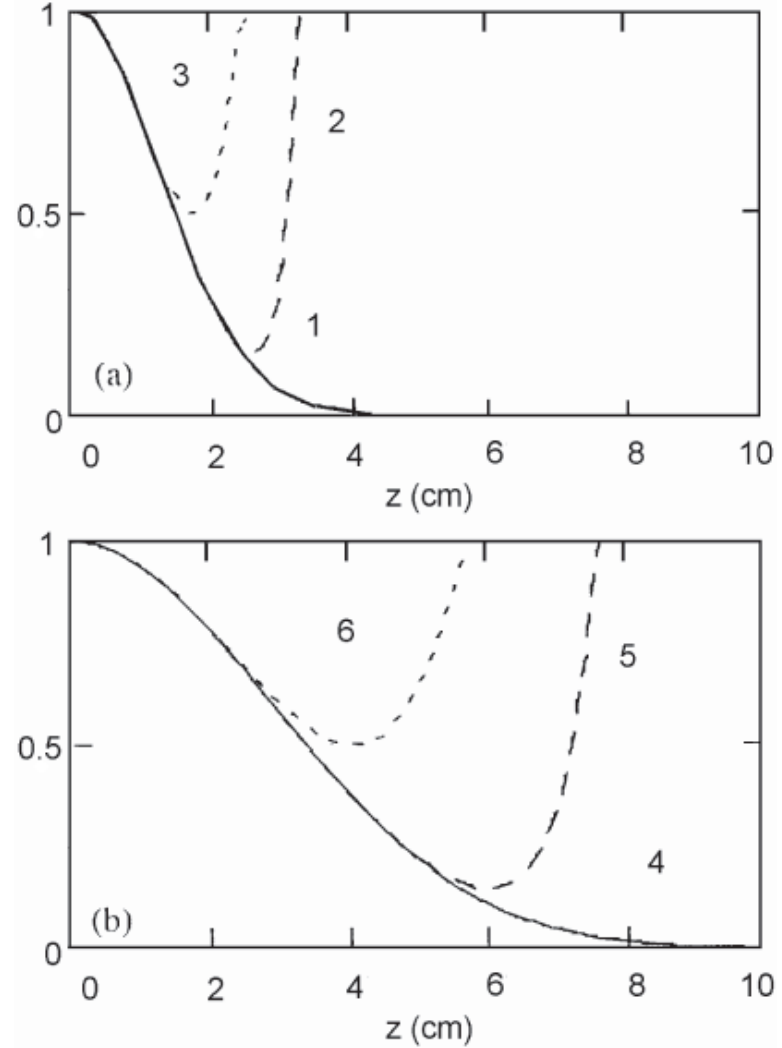

Fig. 2. Graphs representing convergence of $\exp \left(-3 z^{2} / c^{2}\right)$ and its approx function (30) with various nlast and c values: 1. $\exp \left(-3 z^{2} / c^{2}\right), c=3$,

2. $\operatorname{approx}(z, c$, nlast), $c=3$, nlast $=6 ; 3 . \operatorname{approx}(z, c$, nlast $)$,

$c=3$, nlast $=2 ; 4$. $\operatorname{approx}(z, c$, nlast $), c=7 ; 5 . \operatorname{approx}(z, c$, nlast $)$, $c=3$, nlast $=6 ;$ 6. $\operatorname{approx}(z, c$, nlast $), c=7$, nlast $=2$

In general, a source of given c and plates with large thickness, require a high nlast parameter which makes the whole calculation longer. For example, analysing a plate with thickness $\mathrm{g}=5 \mathrm{~cm}$, penetrated by a "double ellipsoid configuration of source" with $\mathrm{c}=2 \mathrm{~cm}$, the approx function must be used with nlast $=13$ (or larger) and is equal to:

$$
\begin{gathered}
\text { approx }=1-\frac{3}{c^{2}} \cdot z^{2}+\frac{9}{\left(2 \cdot c^{4}\right)} \cdot z^{4}-\frac{9}{\left(2 \cdot c^{6}\right)} \cdot z^{6}+\frac{27}{\left(8 \cdot c^{8}\right)} \cdot z^{8}-\frac{81}{\left(40 \cdot c^{10}\right)} \cdot z^{10} \ldots+ \\
+\frac{81}{\left(80 \cdot c^{12}\right)} \cdot z^{12}-\frac{243}{\left(560 \cdot c^{14}\right)} \cdot z^{14}+\frac{729}{\left(4480 \cdot c^{16}\right)} \cdot z^{16}-\frac{243}{\left(4480 \cdot c^{18}\right)} \cdot z^{18} \ldots+ \\
+\frac{729}{\left(44800 \cdot c^{20}\right)} \cdot z^{20}-\frac{2187}{\left(492800 \cdot c^{22}\right)} \cdot z^{22}+\frac{2187}{\left(1971200 \cdot c^{24}\right)} \cdot z^{24}-\frac{6561}{\left(25625600 \cdot c^{26}\right)} \cdot z^{26}
\end{gathered}
$$

So, a Fourier transformation for the z variable can be easily found:

$$
\int_{0}^{\mathrm{g}}\left[\cos (\mathrm{r} \cdot \mathrm{z})+\frac{\alpha_{0}}{\lambda \mathrm{r}} \sin \left(\mathrm{r} \cdot \mathrm{z}_{0}\right)\right] \cdot \operatorname{aprox}(\mathrm{z}, \mathrm{c}, \text { nlast }) \mathrm{dz}
$$

The integral equation (32) is computed with the use of computer symbolic calculation. The result is usually very long. This expression is signed as $\mathrm{E}_{\mathrm{i}}^{1}$ to perform the rest operations.

Through execution the reverse transformation for specified modules for $\mathrm{x}_{0}, \mathrm{y}_{0}, \mathrm{z}_{0}$ in the same way as previously for C-I-N $\mathrm{H}-\mathrm{S}$ model the temporary temperature field generated by a pulsed "double ellipsoid configuration of source" is finally achieved as follows: 


$$
\begin{aligned}
& \mathrm{T}=\left(\frac{\mathrm{q} \cdot \mathrm{f}_{\mathrm{f}} \cdot 3 \sqrt{3}}{\pi \cdot \sqrt{\pi} \frac{\lambda}{\alpha} \mathrm{c}_{\mathrm{f}} \sqrt{\left(12 \cdot \alpha \cdot \mathrm{t}+\mathrm{a}_{\mathrm{f}}^{2}\right)\left(12 \alpha \mathrm{t}+\mathrm{b}_{\mathrm{f}}^{2}\right)}} \cdot \exp \left[-\left(\frac{\mathrm{x}_{0}^{2}}{4 \alpha \mathrm{t}+\frac{1}{3} \mathrm{a}_{\mathrm{f}}^{2}}+\frac{\mathrm{y}_{0}^{2}}{4 \alpha \mathrm{t}+\frac{1}{3} \mathrm{~b}_{\mathrm{f}}^{2}}\right)\right]\right)+ \\
& +\left(\frac{q \cdot f_{r} \cdot 3 \sqrt{3}}{\pi \cdot \sqrt{\pi} \frac{\lambda}{\alpha} c_{r} \sqrt{\left(12 \cdot \alpha \cdot t+a_{r}^{2}\right)\left(12 \alpha t+b_{r}^{2}\right)}} \cdot \exp \left[-\left(\frac{x_{0}^{2}}{4 \alpha t+\frac{1}{3} a_{r}^{2}}+\frac{y_{0}^{2}}{4 \alpha t+\frac{1}{3} b_{r}^{2}}\right)\right]\right) \text {. } \\
& \cdot \sum_{i=1}^{\infty} \mathrm{B}_{\mathrm{i}} \mathrm{C}_{\mathrm{i}} \mathrm{E}_{\mathrm{i}} \cdot \exp \left(-\alpha \cdot \mathrm{r}_{\mathrm{i}}^{2} \cdot \mathrm{t}\right)
\end{aligned}
$$

Using an additivity method, we may achieve the summary temperature field generated by moving heat source as follows: stationary co-ordinates system:

$$
\begin{aligned}
& \mathrm{T}\left(\mathrm{x}_{0}, \mathrm{y}_{0}, \mathrm{z}_{0}\right)=\int_{0}^{\mathrm{t}}\left(\frac{\mathrm{q} \cdot \mathrm{f}_{\mathrm{f}} \cdot 3 \cdot \sqrt{3} \mathrm{dt}}{\pi \cdot \sqrt{\pi} \cdot \frac{\lambda}{\mathrm{a}} \cdot \mathrm{c}_{\mathrm{f}} \cdot \sqrt{\left(12 \cdot \mathrm{a} \cdot\left(\mathrm{t}-\mathrm{t}^{\prime}\right)+\mathrm{a}_{\mathrm{f}}^{2}\right] \cdot\left[12 \cdot \mathrm{a} \cdot\left(\mathrm{t}-\mathrm{t}^{\prime}\right)+\mathrm{b}_{\mathrm{f}}^{2}\right]}} .\right. \\
& \cdot \exp \left[-\left(\frac{\left(\mathrm{x}_{0}-\mathrm{v} \cdot \mathrm{t}^{\prime}\right)^{2}}{4 \cdot \mathrm{a} \cdot\left(\mathrm{t}-\mathrm{t}^{\prime}\right)+\frac{1}{3} \mathrm{a}_{\mathrm{f}}^{2}}+\frac{\mathrm{y}_{0}^{2}}{4 \cdot \mathrm{a} \cdot\left(\mathrm{t}-\mathrm{t}^{\prime}\right)+\frac{1}{3} \mathrm{~b}_{\mathrm{f}}^{2}}\right)\right]+\frac{\mathrm{q} \cdot \mathrm{f}_{\mathrm{r}} \cdot 3 \cdot \sqrt{3} \mathrm{dt}^{\prime}}{\pi \cdot \sqrt{\pi} \cdot \frac{\lambda}{\mathrm{a}} \cdot \mathrm{c}_{\mathrm{r}} \cdot \sqrt{\left(\left[12 \cdot \mathrm{a} \cdot\left(\mathrm{t}-\mathrm{t}^{\prime}\right)+\mathrm{a}_{\mathrm{r}}^{2}\right] \cdot\left[12 \cdot \mathrm{a} \cdot\left(\mathrm{t}-\mathrm{t}^{\prime}\right)+\mathrm{b}_{\mathrm{r}}^{2}\right]\right)}} . \\
& \left.\cdot \exp \left[-\left(\frac{\left(x_{0}-v \cdot t^{\prime}\right)^{2}}{4 \cdot a \cdot\left(t-t^{\prime}\right)+\frac{1}{3} a_{r}^{2}}+\frac{y_{0}^{2}}{4 \cdot a \cdot\left(t-t^{\prime}\right)+\frac{1}{3} b_{r}^{2}}\right)\right]\right) \cdot \sum_{i=1}^{\infty} B_{i} \cdot C_{i} \cdot E_{i} \cdot \exp \left[-a \cdot r_{i}^{2} \cdot\left(t-t^{\prime}\right)\right] \\
& \text { - moving co-ordinates system: } \mathrm{x}=\mathrm{x}_{0}-\mathrm{vt}, \mathrm{y}=\mathrm{y}_{0}, \mathrm{z}=\mathrm{z}_{0} \text { : } \\
& T(x, y, z)=\int_{0}^{t}\left(\frac{q \cdot f_{f} \cdot 3 \cdot \sqrt{3} d t}{\pi \cdot \sqrt{\pi} \cdot \frac{\lambda}{a} \cdot c_{f} \cdot \sqrt{\left.\left(112 \cdot a \cdot\left(t-t^{\prime}\right)+a_{f}^{2}\right] \cdot\left[12 \cdot a \cdot\left(t-t^{\prime}\right)+b_{f}^{2}\right]\right)}} .\right. \\
& \cdot \exp \left[-\left(\frac{\left[\mathrm{x}+\mathrm{v} \cdot\left(\mathrm{t}-\mathrm{t}^{\prime}\right)\right]^{2}}{4 \cdot \mathrm{a} \cdot\left(\mathrm{t}-\mathrm{t}^{\prime}\right)+\frac{1}{3} \mathrm{a}_{\mathrm{f}}^{2}}+\frac{\mathrm{y}^{2}}{4 \cdot \mathrm{a} \cdot\left(\mathrm{t}-\mathrm{t}^{\prime}\right)+\frac{1}{3} \mathrm{~b}_{\mathrm{f}}^{2}}\right)\right]+\frac{\mathrm{q} \cdot \mathrm{f}_{\mathrm{r}} \cdot 3 \cdot \sqrt{3} d \mathrm{t}^{\prime}}{\pi \cdot \sqrt{\pi} \cdot \frac{\lambda}{\mathrm{a}} \cdot \mathrm{c}_{\mathrm{r}} \cdot \sqrt{\left(\left[12 \cdot \mathrm{a} \cdot\left(\mathrm{t}-\mathrm{t}^{\prime}\right)+\mathrm{a}_{\mathrm{r}}^{2}\right] \cdot\left[12 \cdot \mathrm{a} \cdot\left(\mathrm{t}-\mathrm{t}^{\prime}\right)+\mathrm{b}_{\mathrm{r}}^{2}\right)\right.}} . \\
& \cdot \exp \left[-\left(\frac{\left[\mathrm{x}+\mathrm{v} \cdot\left(\mathrm{t}-\mathrm{t}^{\prime}\right)\right]^{2}}{4 \cdot a \cdot\left(t-\mathrm{t}^{\prime}\right)+\frac{1}{3} \mathrm{a}_{\mathrm{r}}^{2}}+\frac{\mathrm{y}^{2}}{4 \cdot a \cdot\left(t-\mathrm{t}^{\prime}\right)+\frac{1}{3} \mathrm{~b}_{\mathrm{r}}^{2}}\right)\right] \sum_{\mathrm{i}=1}^{\infty} \mathrm{B}_{\mathrm{i}} \cdot \mathrm{C}_{\mathrm{i}} \cdot \mathrm{E}_{\mathrm{i}} \cdot \exp \left[-\mathrm{a} \cdot \mathrm{r}_{\mathrm{i}}^{2} \cdot\left(\mathrm{t}-\mathrm{t}^{\prime}\right)\right]
\end{aligned}
$$

where:

$$
\begin{gathered}
E_{i}=\int_{0}^{g}\left(\cos \left(r_{i} \cdot z\right)+\frac{\alpha_{0}}{\lambda \cdot r} \cdot \sin \left(r_{i} \cdot z\right)\right) \cdot \operatorname{approx}(z, c, \text { nlast }) d z \\
\operatorname{approx}(z, c, \text { nlast })=1+\sum_{n=1}^{\text {nlast }} \frac{\left(-\frac{3}{c^{2}} \cdot z^{2}\right)}{\prod_{i=1}^{m} n}
\end{gathered}
$$


Equations (34), (35) have a similar features as previously determined equations (23), (24) for assessment of the temperature fields in both stationary and moving coordinates systems. Both solutions have been given for three dimensional C-I-N and D-E heat source models but with different thermal characteristic. Especially it is obliged to differentiate low or high concentration of energy of heat sources.

Remaining comments concerning manner of solutions of equations (34), (35) and their results are the same as presented previously for equation (23), (24). The employment of method of hybrid account exists also in this case. That is the main purpose of the III part of this work. For further calculations it is chosen the analytic-numerical method.

\section{CONCLUSIONS}

In this paper the new temperature evaluation during welding of a plate with optional thickness is presented. Furthermore, the radiative heat transfer on both surfaces is taken into account. In these calculations we used the following heat source models: cylindrical-involution-normal (C-I-N) and double ellipsoidal configuration of source (D-E). By using the Fourier transformation method, the temperature fields generated by above heat sources in both stationary and moving co-ordinates system are presented. The algebraic forms of analytic solutions presented by equations requires discretising in order to make possible numerical calculation.

\section{NOMENCLATURE}

$\begin{array}{ll}\overline{\mathrm{T}}, \overline{\mathrm{T}}, \overline{\overline{\mathrm{T}}} & \text { - } \\ \mathrm{K}\left(\mathrm{r}, \mathrm{x}_{0}\right), \mathrm{K}\left(\mathrm{r}, \mathrm{y}_{0}\right), \mathrm{K}\left(\mathrm{r}, \mathrm{z}_{0}\right) & - \text { transformations modules for } \mathrm{x}_{0}, \mathrm{y}_{0}, \mathrm{z}_{0}\end{array}$
$\mathrm{R}\left(\mathrm{r}, \mathrm{x}_{0}\right), \mathrm{R}\left(\mathrm{r}, \mathrm{y}_{0}\right), \mathrm{R}\left(\mathrm{r}, \mathrm{z}_{0}\right)$ - reverse transformations modules

$\mathrm{c}_{\gamma} \quad$ - volumetric heat, $\left[\mathrm{J} \mathrm{K}^{-1} \mathrm{~cm}^{-3}\right]$

$\mathrm{c}_{\mathrm{p}}^{\gamma} \quad$ - specific heat, $\left[\mathrm{J} \mathrm{K}^{-1} \mathrm{~kg}^{-1}\right]$

$\rho$ - mass density, $\left[\mathrm{kg} \mathrm{cm}^{-3}\right]$

$\prod_{i=1}^{m} n$ - performs iterated multiplication of $\mathrm{n}$ over $\mathrm{i}=1, . ., \mathrm{m}-1,[\mathrm{~m}]$

$B_{i}, C_{i}$ - values are in agreement with (23a) and (23b)

nlast - natural positive number as large as is necessary to achieve the required approximation

HAZ - Heat affected Zone

C-I-N - Cylindrical-Involution-Normal heat source model

D-E - Double-Ellipsoidal heat source model

HS - Heat Source.

\section{BIBLIOGRAPHY}

1. E. Ranatowski, A. Poćwiardowski: Mathematical Modelling of Weld Phenomena 5, (ed H. Cerjak),pp. 725-742. 2000. London

2. G.A. Korn, T.M. Korn: Mathematics for scientists and engineers. McGraw - Hill Book Company. Copyright for Polish edition by PWN. Part I, pp. 144-146. 1983. Warsaw

3. Benker: Practical use of Mathcad: Solving of Mathematical Problems. Springer-Verlag. Berlin Heidelberg. 1999.

\section{CONTACT WITH THE AUTHOR}

Prof. Ranatowski Eugeniusz Faculty of Mechanical Engineerig, University of Technology and Life Science, Prof. S. Kaliskiego 7 85-763 Bydgoszcz, POLAND e-mail: ranatow@utp.edu.pl 